\title{
Energy Savings by Wireless Control of Speed, Scheduling and Travel Times for Hauling Operation
}

\author{
D. Rylander, J. Axelsson, P. Wallin
}

\begin{abstract}
A Quarry and Aggregate production site consist of sequential production processes and activities to process and produce the output products. Compared to a fixed manufacturing plant, the quarry processes involve mobile machines such as wheel loaders, trucks and articulated haulers and a highly dynamic road infrastructure. Today, the mobile machines are generally not synchronized or controlled towards the overall throughput of the site in real time. This indicates a general improvement potential in increased productivity at quarry sites, but also unsolved challenges for the same reason. Assuming a wireless control system that controls speed and throughput of the different processes and activities, there would be a fuel reduction potential in controlling the mobile machines. This optimization requires models of machine fuel consumption for different applications, velocities and travel times. The main contribution of this paper is the presentation of fuel measurements based on different speeds, site application characteristics and travel times for hauling operation. The fuel measures reveal important aspects regarding how different velocities impact fuel consumption. The results of fuel measurements show a potential in fuel savings of up to $42 \%$ and a typical improvement of $\mathbf{2 0 - 3 0 \%}$ depending on machine speeds, travel times, application and site characteristics. Based on this, some of the applications and challenges in wirelessly controlling machines are discussed.
\end{abstract}

Keywords; Fuel Measurements, Vehicle Control, Hauling optimization, Advanced Driver Assist Systems

\section{INTRODUCTION}

Quarry and Aggregates is an important business that supplies sand, gravels, aggregates, cement and asphalt for all sort of construction projects. Quarries are available all over the world and only in Europe there are over 25000 quarries [1].

The Quarry production process is a process where stone and rock material is extracted from the ground through a drilling/blasting or ripping/hammering activity. The raw material and main supplies is then processed using crushers and screening activities to obtain the target products for delivery to customers. The supply material is usually located at a distance from the production plant which requires transport activities by mobile machines. In addition, mobile machines are also required to sort and balance piles at stockpiling areas and customer loading activities (see Figure

D. Rylander is with the School of Innovation, Design and Engineering, Mälardalen University, Västerås, Sweden (phone:+46 (0)73-902-7509; email: david.rylander@volvo.com).

J. Axelsson is with Mälardalen University, Västerås, Sweden (e-mail: jakob.axelsson@mdh.se).

P. Wallin is with Volvo Construction Equipment, Eskilstuna, Sweden (e-mail: peter.wallin.2@volvo.com)
1). These transporting, moving and loading activities are mainly performed by trucks, articulated haulers and wheel loaders.

For the transport to the crusher activity, it is common that sites have a large amount of unnecessary stops during transport operation due to occasionally narrow meeting areas, and waiting times caused by overcapacities and uneven mass processing capacities [2]. Based on the use of different machine types and performance, the waiting times may occur both at loading of raw material and at unloading at the crusher. In [2], it has been stated that average waiting times may be up to $30 \%$ of the total transport cycle time.

The overcapacity in the transport process compared to the crushing process is easily explained in the need to manage variations in the production rate of different activities. At the quarry site, both the transport and crushing activities vary its capacity over time. The crusher capacity is mainly depending on stone material and its density as well as size. The capacity also depends on the maintenance schedule since the production rate is higher with new components compared to old due to wear and tear. The transport process includes even more dependencies. The main activities are loading, transport and unloading. Loading time is mainly dependent on machine capacity used for loading which may vary; the load capacity utilization also depends on material type and size. Transport and travel time is dependent on several things, where distance is the main factor, but topology, surface conditions, machine speed capacity and machine maintenance also affect the time for transportation. In addition, the site configuration may result in meetings at bottlenecks and required stops during transport, e.g., due to intersections which affect the time needed for the transport. Many of these characteristics change over time due to weather conditions, maintenance schedules, and the fact that the ground is processed which naturally changes the characteristics of the site.

Based on these site characteristics, there is a clear potential in optimizing site throughput by better coordinating

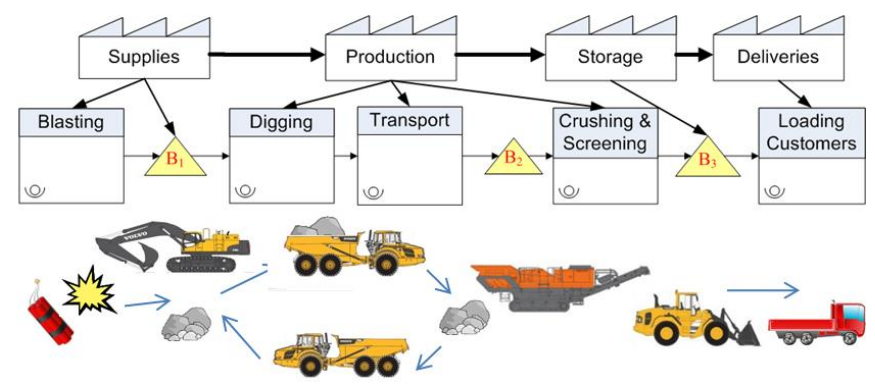

Figure 1. Quarry Production Processes 
machine movements, and this is the focus of our research. Such an optimization would require a site control system that uses wireless communication to control the speed for each machine. In this way, the machines can travel at a more energy efficient speed, and hence with lower fuel consumption, instead of moving at maximum speed and then wait at the destination. However, for the site control system to be able to select the appropriate speed, it requires a model of the fuel consumption characteristics of each machine type, and the construction of such a model is the main research question addressed in this paper.

\section{A. Related work}

Previously, it has been concluded through driving studies that fuel consumption is heavily dependent on driver skills [3]. Fuel consumption can increase as much as two to three times when comparing a novel and expert driver under similar driving conditions, machines and applications. Research has also concluded that machine specific fuel characteristics such as idling fuel consumption of dump trucks can be optimized using trajectories and prediction algorithms [4].

In addition, there have been numerous simulations presented where different approaches to controlling the driveline through terrain and trajectory predictions and intelligent gear shifting are investigated [5]. For optimal productivity it has been derived how total cost of ownership is affected by having the right size and type of machine for a specific task and site [6]. But anyhow there is a need to handle fluctuations in the production and need of site overcapacities to manage deviations in the production chain. In addition, manufacturers supply machine and motor specific fuel characteristics for driving cycles [7] but not field trials in application usage where these data have been validated.

On a technical basis the potential of controlling vehicles at a quarry site using wireless short range communication has successfully been demonstrated for sufficient coverage and latency in smaller quarries [8].

What is lacking from current research is models showing how real world energy consumption of applicable machines are affected by stop-and-go driving, as well as different speed profiles and consequently changes in travel times in quarry operation. To be able to control the vehicles wirelessly, such models need to be validated.

The contribution of this paper is the presentation of how the driving characteristics of a hauler affect fuel consumption based on real world measurements in a quarry application. The characteristics include variation in speed and effects of unmotivated and non-value adding stops during operation. Further the paper evaluates potential fuel savings based on different velocities, site application characteristics, and travel times for an application relevant machine. In addition the paper discusses typical applications where this knowledge can be utilized.

\section{METHOD}

To investigate this problem an iterative data collection approach has been used. The empirical data on which the results build has been collected through analysis of drive cycles with an articulated hauler. The drive cycles were performed in a terrain that is comparable to general quarry operation. The empirical study contains iteratively performed test cases to achieve aggregated relevant values. To obtain comparable results, a single machine of type Volvo A40FS has been used for all tests. There may be differences towards other brands, types, and sizes which are not counted for in this paper. The tests have been conducted in two test site configurations, with different test characteristics. The first configuration was a highly controlled environment at a flat surface to measure how the velocity and travel times affect fuel consumption along with how extra stops during transport affect overall fuel consumption. In addition a second configuration has been used where three professional drivers conducted speed tests in rough terrain to see the applicability of the results in real conditions and understand the dependencies from the drivers.

\section{DATA COLLECTION}

To collect the data required to be able to create a fuel model for one vehicle, the different dependencies need to be identified. As previous research has concluded, driver style affect fuel consumption. Driver style affects usage of brake, throttle and steering, which affect acceleration and retardation as well as path. Acceleration and retardation affect speed and usage of obtained energy. Path can be referred to as a distance in a terrain which has a specific topology and surface which affect the traction and friction. In addition, external factors such as temperature and weather are factors that affect fuel consumption. While measuring fuel consumption, the method used is important to understand the limitations and accuracy of the data obtained.

To limit the dependencies in the data collected, measurements were performed in two main data collection configurations in which different factors were observed while others were minimized. The main aspects of this study is how speed, driver behavior and travel time affect fuel consumption which is the focus of the collection configurations. The aspects of speed travel time and amount of stops performed during the operation is observed in Configuration 1. General driver dependencies and vehicle speed is observed in Configuration 2.

The measuring tool for fuel consumption was mainly continuous data logging from the in-vehicle Controller Area Network (CAN) bus during runtime of the machine. In addition, manual time watch, trip logger and the satellite based global positioning system (GPS) were used to measure time and distance. In the controlled environment at the flat track an additional fuel tank was used to measure and verify the fuel consumption in a highly accurate way, see Figure 2. The additional measuring fuel tank was connected between the engine and the normal fuel tank. It was equipped to exactly measure the actual fuel consumed. This measured data was logged after each lap. Worth mentioning is that during the analysis there were no major differences or 
characteristics deviations observed between the two measuring methods.

To minimize disturbance factors in the test environment the machine was pre-heated before any data was collected. A factor that may affect the results was the human controlled difference in acceleration and retardation during cycles. This was approached through awareness of the issue and minimized through consideration in the trial by the drivers. Wear of machine and tires was considered as negligible due to the limited use of the machine in the trials. In addition each data configuration was performed during two subsequent days with cloudy weather at 8 to 15 degrees Celsius.

\section{A. Data Collection Configuration 1}

As a precise measurement of how the machine operation influences fuel consumption, independent of driver skills, field tests on simple straight test track with good road conditions were conducted. The study in this controlled environment was conducted in a $797 \mathrm{~m}$ flat oval test track using a single professional driver. The test result assists in determining the fuel consumption characteristics for different average velocities and stop-and-go activities. Each cycle was driven 5 times for each gear, to the predefined test cases at $1500 \mathrm{rpm}$. The hauler used had gears ranging from 1-9, where 9 is the highest gear and therefore also highest speed. $1500 \mathrm{rpm}$ was chosen since it is a stable rpm in between gear changes in the automatic gearbox of the machine used. In this way, the machine did not alter gear during the measurements, which was expected to affect the results and create uncertainty in the measurements. The measurement of each lap started and stopped at speed 0 with the machine standing still on the same geographical position for each lap. The gear during driving did not alternate except at acceleration and retardation phase. Acceleration to lap speed and retardation to 0 was done at machine max (full) capacity; basically accelerate as fast as possible to test max gear and full break for retardation at finish line. Each test case of driving, both with and without stops, was done without load and with 38 ton load maximal capacity, respectively. When

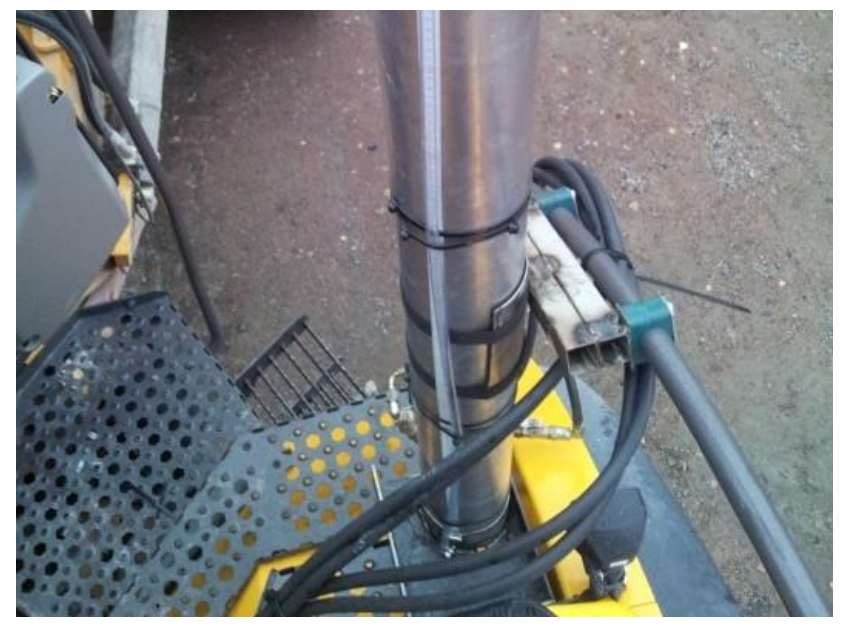

Figure 2. Fuel measurement tank connected between hauler fuel tank (override) and engine the scenario included stops, the stops were evenly distributed over the test track distance.

\section{B. Data Collection Configuration 2}

To create a reference to naturalistic driving conditions and compare different driver styles, a study in rough terrain was performed. The track had a total distance of 2250 meters of rough terrain and an elevation of up to 50 meters, similar to the terrain characteristics at a quarry site. Three skilled drivers drove 4 laps with max performance and speed, and 4 laps with eco driving instructions with a fully loaded articulated hauler. The measurements started and stopped at speed 0 , with the machine standing still. The eco driving instructions were to avoid breaking, avoid using throttle max, and not at all use throttle during downhill/descending

\section{ANALYSIS}

In measurement Configuration 1, data was collected in five driving scenarios:

- Unloaded driving

- Unloaded driving with 1 stop per lap

- Fully loaded driving

- Fully loaded driving with 1 stop per lap

- Fully loaded driving with 2 stops per lap

The measured result in terms of fuel consumption for the five driving scenarios when gear 7 and 9 was used is presented in Figure 3. The maximum fuel consumption is defined as $100 \%$ and determined by the most fuel consuming driving style. The other values presented in the figure are fuel consumption decrease in percentage related to the maximum value.

In Figure 3 it can be seen that a fully loaded machine has an increased fuel consumption of roughly $20-25 \%$ compared to an unloaded machine. In addition, each stop per lap increase the fuel consumption with $10-15 \%$.

Comparing the fuel consumption of the most consuming

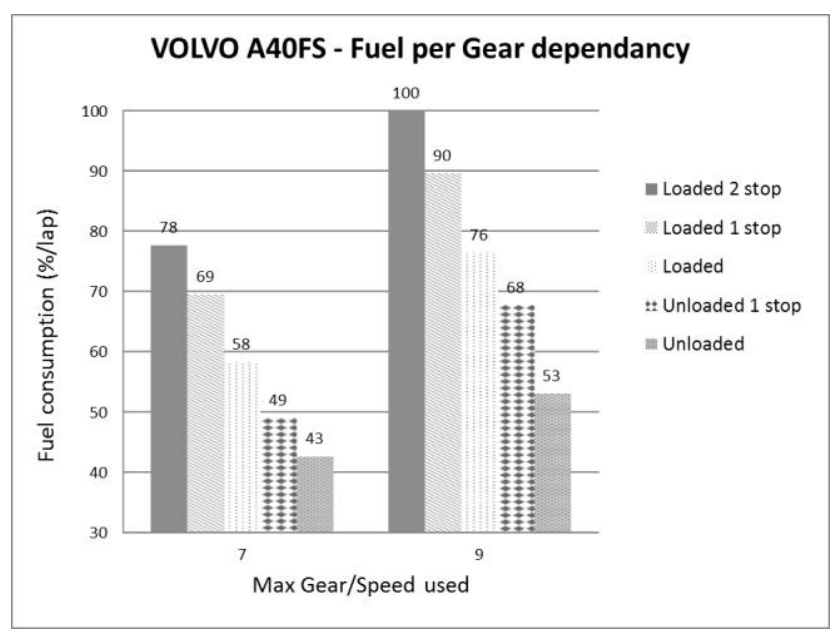

Figure 3. Fuel consumption relation between top gear, weight and stops 


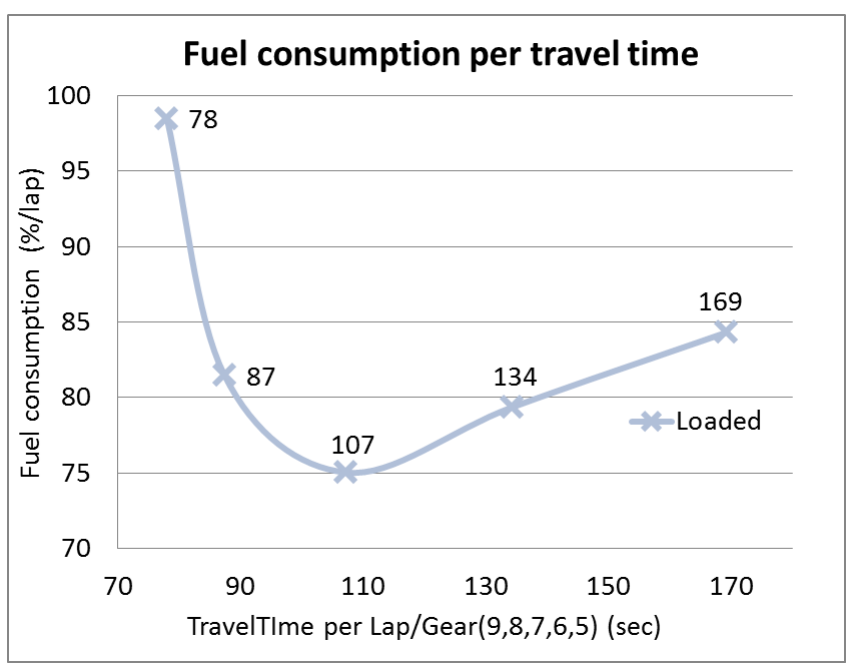

Figure. 4. Fuel consumption per travel time for each lap

with the least consuming style and speed with full load shows a decrease in fuel consumption by $42 \%$. The difference is the 2 unnecessary stops per lap and maximum speed allowed between the styles.

The measures per gear do not necessarily represent a linear relation to the speed and travel time for a lap, since the speed difference between gears is not required to be the same, e.g., comparing gear 5 to 6 and 8 to 9 . From the same measurements in Configuration 1, Figure 4 show the relation of fuel consumption based on average travel times for each lap with the fully loaded machine, without unnecessary stops.

The graph in Figure 4 shows that while increasing the lap time from $78 \mathrm{~s}$ to $107 \mathrm{~s}$ by changing maximum gear from 9 to 7 , resulting in a decrease of average speed from 37 to 27 $\mathrm{km} / \mathrm{h}$, the decrease in fuel consumption was nearly $25 \%$.

In addition, reductions were only observed from gear 9 to gear 7. When speed was decreased even more, using only gears below 7 the fuel consumption increased. From the results obtained in this study it seems that there is an optimal speed and gear to use for this machine while on flat surface. and unloading by an average of $30 \%$ of the operating time for haulers. In Figure 4 the increase of travel time by $37 \%$ from $78 \mathrm{~s}$ at top speed to $107 \mathrm{~s}$ would decrease the fuel consumption from start to stop by nearly $25 \%$. Since this time may anyhow be spent waiting, such a decrease would not affect the production rate of the site if it could be coordinated and monitored continuously.

In Figure 5, the fuel measurements per driver in rough terrain are presented from data collection in Configuration 2. Since the driving was done without continuous control, the operators made individual deviations from lap to lap. Analyzing the graph indicates a clear pattern towards a change in fuel consumption per cycle. On the eco-driving laps, the overall speed of the machine decreased, leading to increased cycle times, but at the same time the fuel consumption decreased significantly. This shows that the speed of a vehicle has a direct influence on the fuel consumption over a defined distance. Analyzing the measurements shows that a difference of nearly $30 \%$ in the rough terrain was accomplished by decreasing travel times and speed accordingly. The savings could be seen both uphill and downhill, even though the largest saving was downhill.

Concluding the measurement analysis it can be determined that the amount of stops per $\mathrm{km}$ add approximately $10 \%$ fuel consumption per stop. The decrease in lap travel time from highest gear and speed to optimal gear, the speed changed from $78 \mathrm{~s}$ to $107 \mathrm{~s}$ with the fully loaded hauler. This is an increase in travel time of $37 \%$ which resulted in decreased average fuel consumption per lap of almost $25 \%$. Comparing these figures to the test cases in terrain with several drivers, the characteristics are comparable, even though the variation was significant between the laps. The fastest lap had a travel time of $264 \mathrm{~s}$, when comparing the fuel consumptions with the lap with the lowest fuel consumption which had a travel time of $347 \mathrm{~s}$ the later consumed $25 \%$ less fuel. This increase in travel time is $31 \%$. The two test configurations did not show exactly the same results but the tendency is the same in terms of fuel savings of about $20-30 \%$ with increased travel time of between $30-37 \%$.

In [2], it was described that waiting can occur at loading

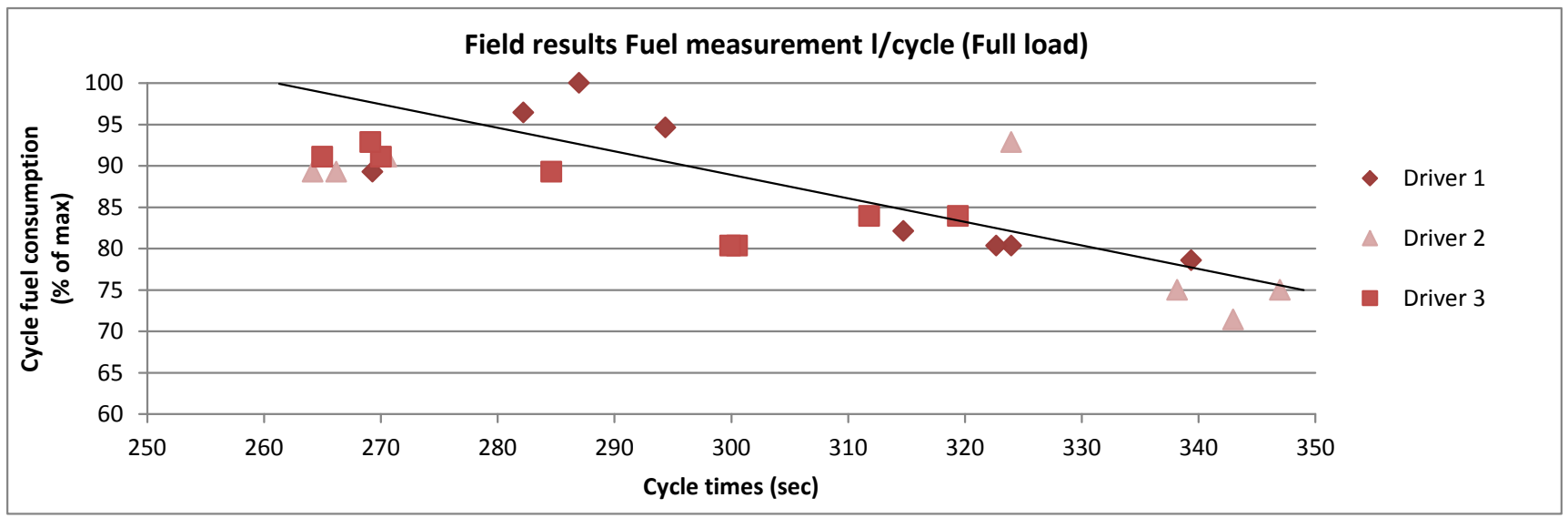

Figure 5. Fuel measurements in lap time for each driver in rough terrain 


\section{DISCUSSION}

In [2] it was shown that quarry sites can have up to $30 \%$ wastes in waiting times and unnecessary stops. In addition, [8] shows that communication coverage can be obtained with wireless ad-hoc communication technologies between mobile machines in a quarry environment. This paper indicates that using wireless communication to address the need for scheduling to minimize waste would potentially be able to save up to $42 \%$ fuel in poorly optimized sites. The potential assumes current site characteristics including several stops per lap and high speed driving with a large amount of waste in waiting time between worst and best case.

Due to this a quarry operation can be assumed to have a potential to be optimized based on designing the processes' and machines' capacities towards the overall throughput in the system. This should decrease the waste in a poorly optimized operation. There are many sources of variability in production: distance and topology (height difference) from which loading and unloading occur; maintenance stops for individual machines; occasional personnel losses; and machines having decreased capacity for different reasons. In addition, the sites may want to have spare capacity in the production chain to be able to manage these production rate variations and deviations. Due to this, the capacity needs to be optimized with respect to throughput in real-time and bottle neck avoidance independently of how well the machines are designed for the overall process. The control can then be done by lowering capacity and speed of the processes which have an overcapacity, or in some cases, lower the speed but keep the same capacity.

In [2], it was concluded that waiting time often occurs in real world quarry operation as well as unnecessary stops due to narrow roads. The fuel measurements in this paper show that it is not always better to decrease speed to improve fuel efficiency. There is a gear and speed combination that is optimal and it may depend on the individual characteristics of the machine. Stops during operation which do not add value to the operation are always bad for fuel efficiency and should be avoided. These areas can be referred to as bottlenecks, which can be curves, intersections and narrow road links in which more than one vehicle cannot pass simultaneously. Therefore the logistics of avoiding stops needs to be constantly scheduled and rescheduled based on the speed of the meeting machines. But operation at very low speed may affect the overall consumption negatively while trying to avoid stops which constantly need to be monitored.

In a real-world operation, the applicability of a control system will depend on the current operation and site layout as well as vehicle fleet configuration. If a site has bad conditions with several bottlenecks, vehicles are likely to be required to stop to meet other vehicles working at the site. Such a site would not only have the stop and go incidents; it can also be assumed that travel times at the site fluctuate due to the same reason [2]. These fluctuations create an uneven production rate and material flow at the site, which then either requires an overcapacity of the process or maximizing the velocities of the vehicles to make sure they are not too late to deliver material for the next process, causing production downtime which decrease production rate.
Regarding the fuel measurements presented in this paper, such a site would have a potential to save up to $42 \%$ fuel, comparing worst case (two stops at full speed compared to optimal speed without stops, Figure 3) with optimal case, in which the production is controlled. A site without narrow areas and consequently without unnecessary stops may have a potential of controlling velocities towards throughput and does normally not have the same fluctuations in travel times. What will affect though is the site configuration in number of loading and unloading areas to manage for each vehicle. When several such areas are available it can be expected to cause fluctuations in travel time and uneven waiting times due to the uneven capacities of the hauling. The uneven capacities are common due to that sites often consist of haulers from different brands, different sizes and different performance in speed capacity.

To be able to avoid stops due to narrow roads, the system requires awareness of where these areas are located and where they are not. Such awareness requires some sort of geographical map over paths and widths. Such a geographical representation is common for road vehicles, but not for commercial construction sites such as quarries. Instead the construction sites are dynamic and path attributes such as width may change during a workday. Therefore, a dynamic continuous logging of paths by each vehicle and the events such as stops during operation, is required.

Another requirement is the need of reliable communication. The adjustments of speed would be based on need of arrival due to site throughput to avoid waiting, but also prediction of meetings and matching to optimal paths of more than one machine. This demands common knowledge about not only speed and heading but also destinations of all vehicles to match to certain paths available, to be able to conclude optimal speed and meeting areas. Since the machines seem to have a nonlinear speed profile, the site control system needs to know the machine speed profile and characteristics for each machine in the system to be able to calculate optimal speed for each individual machine. Obviously, such an optimization becomes even more difficult at sites with multiple loading and unloading areas to be able to predict each activity's production speed, and the optimal time of arrival for each vehicle.

\section{CONCLUSIONS}

From a fuel consumption perspective, the variation of speed and unnecessary stops can decrease fuel consumption with up to $42 \%$, when comparing optimal gear usage without unnecessary stops to driving full speed and having two stops per lap. When comparing fuel consumption without stops, the consumption decreased with $24 \%$ while driving fully loaded at optimal speed, compared to full speed.

In regard to these measurements, it can be concluded that a decrease in travel time can affect fuel consumption significantly. However, it was also shown that decreasing speed, at least on a flat surface, does not necessarily mean decreased fuel consumption, but instead the opposite occurs at lower speeds. What is seen in the fuel measurement study is that for a $797 \mathrm{~m}$ flat track, the machine had an optimal 
max speed to minimize fuel consumption. This is probably the case for most vehicles, but with variations depending on the make and model of machine.

Another important factor to consider based on the study is the number of unnecessary stops that occur due to narrow tracks and bottlenecks. This implies the need of a site control system based on accurate real-time communication to exchange positions, travel times, geographical representation and other relevant data to be able to control the speed of each vehicle towards the throughput of the site. Since sites may have several unloading and loading areas, the information about each vehicle's destination is crucial for the scheduling applications.

Comparing the study presented in this paper towards a real world environment, it can be concluded that a number of characteristics affect the potential for optimizing the site such as;

- Site layout with the number of loading, unloading areas and bottlenecks

- Machines, both type and the number of machines used

- Capacity and capacity difference in the different processes

- Travel distances and path/road characteristics, such as width, topology and friction

\section{FUTURE WORK}

This paper highlights the potential reductions in fuel consumption by coordinating the hauling activities at a quarry site. The paper further discusses the implications of implementing a control system. Future work will therefore develop and evaluate solutions for this purpose. One of the main challenges is how communication performance, coverage, latencies and throughput at a remote location influence the system and optimization algorithms. Different system designs and intelligence strategies, e.g., centralized vs. decentralized intelligence, may manage the issues differently and this needs to be addressed and further analyzed.

Another important challenge is how to manage road maps, paths and travel times. This is of particular interest for a quarry site since the actual operation of processing ground rock material changes the road and site topology over time, so that one road that exists today may not exist tomorrow. This fact can be expected to influence the optimization and estimation of travel times severely for mobile machines. A benefit of the dynamic roads is that with detailed analysis of the site it might even be possible to construct the roads in a way that fuel efficiency can be improved even further.

The third challenge which is of great importance is the communication data protocol strategy and attributes to exchange information between machines performing optimized hauling operations, and this will also be further elaborated in the future. The applications that can optimize speed towards optimal time of arrival to the destination to unload or load should be carefully scheduled based on machine fuel profiles, site terrain, distances, travel time and meetings with the other machines. These scheduling algorithms and components for these applications will be further developed in the future.

Further, it would be an interesting complement to perform fuel measurements for other machine types and brands. In addition, the fuel measurements could be improved with more test cases of how the machine performs in different terrain profiles in terms of fuel consumption, e.g., uphill and downhill.

\section{ACKNOWLEDGMENT}

We would like to thank all colleagues at Volvo assisting in the setup and execution of the trials, especially Mr. Mårten Andersson and Mr. Erik Uhlin for their valuable support. This research is supported by the Knowledge Foundation (KKS) through ITS-EASY, an Industrial Research School in Embedded Software and Systems, affiliated with Mälardalen University, Sweden.

\section{REFERENCES}

[1] UEPG. (2012). European Aggregates Association. Available: http://www.uepg.eu/

[2] D. Rylander and J. Axelsson, "LEAN METHOD TO IDENTIFY IMPROVEMENTS FOR OPERATION CONTROL AT QUARRY SITES," presented at the ISARC Conference, Montreal, 2013.

[3] B. Frank, L. Skogh, R. Filla, and M. Alaküla, "On increasing fuel efficiency by operator assistant systems in a wheel loader," presented at the 2012 International Conference on Advanced Vehicle Technologies and Integration (VTI 2012), Changchun, China, 2012.

[4] B. Saerens, "Minimization of the fuel consumption of a gasoline engine using dynamic optimization," Applied energy, vol. 86, p. $1582,2009$.

[5] J. Fu and G. Bortolin, "Gear Shift Optimization for Off-road Construction Vehicles," Procedia-Social and Behavioral Sciences, vol. 54, pp. 989-998, 2012.

[6] E. Uhlin, "Microsimulation of Total Cost of Ownership in Quarries," in 17 th International Conference of Hong Kong Society for Transport Studies, 2012.

[7] C. E. Volvo. (2008, Volvo GPPE Performance Manual.

[8] S. Vernersson, E. Kalpaxidou, and D. Rylander, "Evaluation of Wireless Short-Range Communication Performance in a Quarry Environment," presented at the International Conference on Connected Vehicles and Expo (ICCVE), Las Vegas, USA, 2013. 